\title{
Couche archéologique, sol archéologique et films de suie pariétaux : une approche micro-chronologique de l'occupation des cavités
}

\section{Archaeological layer, archaeological floor and parietal soot films: a micro-chronological approach to understanding occupations in caves}

\author{
Ségolène Vandevelde ${ }^{1}$, Jacques É. Brochier ${ }^{2}$, Ludovic Slimak ${ }^{3}$ \\ ${ }^{1}$ Université Paris 1 - Panthéon-Sorbonne, UMR 7041 ArScAn, équipe Archéologies environnementales, Paris, \\ France, segolene.vandevelde@univ-paris1.fr \\ ${ }^{2}$ Aix-Marseille Univ, CNRS, Minist Culture, LAMPEA UMR 7269, Aix-en-Provence, France, \\ jacques.brochier@univ-amu.fr \\ ${ }^{3}$ Université Toulouse Jean Jaurès, UMR 5608 CNRS, TRACES, Toulouse, France, slimak@univ-tlse2.fr
}

RÉSUMÉ. Des traces de suie, témoignant d'activités humaines passées, sont parfois remarquées sur les parois des cavités, parfois également à l'intérieur de spéléothèmes. Ces dépôts, qui résultent de feux anthropiques, s'avèrent être un matériau particulièrement adapté aux études micro-chronologiques à très haute résolution. L'analyse microscopique d'encroûtements carbonatés de parois d'âges variés (Paléolithique moyen et supérieur) montre qu'ils ont conservé la trace de multiples occupations qu'il est possible de mettre en relation avec les unités archéologiques reconnues à la fouille. Les NMO (Nombre Minimum d'Occupations), généralement élevés, qui correspondent à chaque unité archéologique, attestent du caractère cumulatif de ces dernières. Chaque niveau enregistre alors un nombre d'occupations différent d'une unité à l'autre et illustre des rythmicités qui lui sont propres. Les perspectives de recherche sur les dépôts de suie sont diversifiées et laissent entrevoir la possibilité d'une étude de la mobilité des groupes humains passés suivant des résolutions jusqu'alors inégalées. On est de plus en droit de s'interroger sur le concept de «sol archéologique » et de «palimpseste » appliqué aux études spatiales en grotte et sous abri-sousroche.

ABSTRACT. Traces of soot reflecting past human activities are sometimes observed on the ceilings and walls of caves and rock shelters, sometimes also inside speleothems. These deposits, which result from anthropogenic fires, are proving to be a particularly suitable material for very high-resolution micro-chronological studies. Microscopic analysis of carbonate crusts from walls of various ages (Middle and Upper Palaeolithic) shows that they preserve traces of multiple occupations that can be linked to the archaeological units identified during the excavation. The generally high MNOs (Minimum Number of Occupations), which correspond to each archaeological unit, attest to the cumulative nature of the latter. Each level records a different number of occupations from one unit to another and presents particular rhythmicity. The research potential for soot deposits are diverse and suggest the possibility of studying the mobility of past human groups with previously unrivalled temporal resolution. Concerns about the concept of "archaeological floor" and "palimpsest" as applied to spatial studies in caves and under shelters/under rock is also developed.

MOTS-CLÉS. Micro-chronologie, carbonates, suie, unités cumulatives, palimpseste, sol archéologique.

KEYWORDS. Micro-chronology, carbonates, soot, cumulative units, palimpsest, archaeological floor.

\section{Introduction}

La mention de la présence de suie et/ou de charbon de bois sur les parois des cavités, ou dans des formations de type spéléothème, est rare dans la littérature archéologique quand il ne s'agit pas de 
celle de leur emploi comme pigments noirs utilisés dans l'art rupestre ${ }^{1}$. Quelques cas ont cependant été signalés de par le monde.

Concernant les parois de cavités, des traces récentes ont par exemple été remarquées en Amérique à la Cueva del Diablo au Guatemala (Ishihara-Brito \& Guerra, 2012) ou dans des grottes à salpêtre des États-Unis (Duncan, 1997), en Eurasie, à la grotte de Shanidar en Irak (Solecki, 1979), dans des abris pastoraux en Turquie (Gonzales \& Martinez, 1998), ou au Höhlen am Sirgenstein en Allemagne (Striebel, 1996). Il existe également des dépôts carbonés beaucoup plus anciens, comme ceux de l'Aven de la Mort de Lambert (Lachenal \& Rucker, 2009) et de la Grotte Chauvet (Clottes et al., 1995) en France. En Océanie, les parois de certaines grottes ont été recouvertes de suie pour créer un fond noir en préalable à la réalisation de peintures : c'est le cas à Aibura Cave en Nouvelle-Guinée (White, 1965) et à Beswick Creek Cave en Australie (Macintosh, 1952). Voir Vandevelde et al. (2018) pour d'autres exemples.

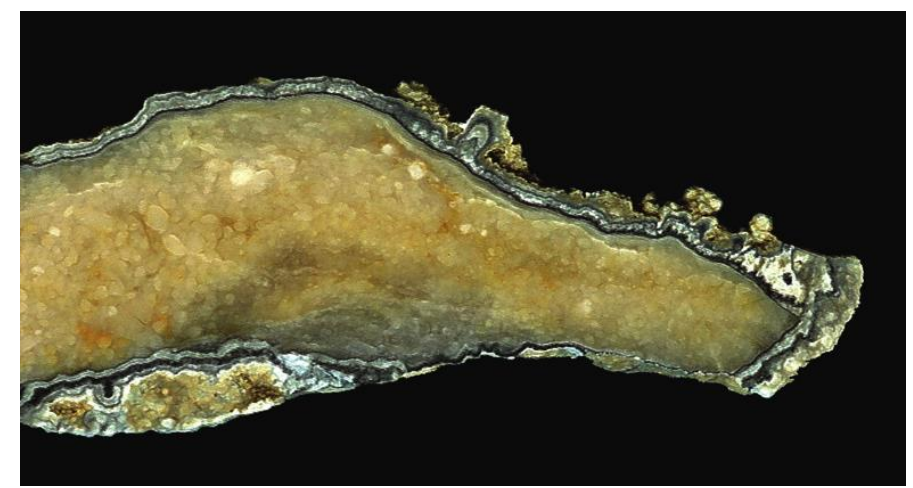

Figure 1. Section polie d'une plaquette provenant de la Grotte Mandrin, couche C. Remarquer le petit nombre de films de suie discernables et, sur la partie droite du fragment, le développement d'une seconde génération d'encroûtement carboné après détachement d'une partie de la plaquette.

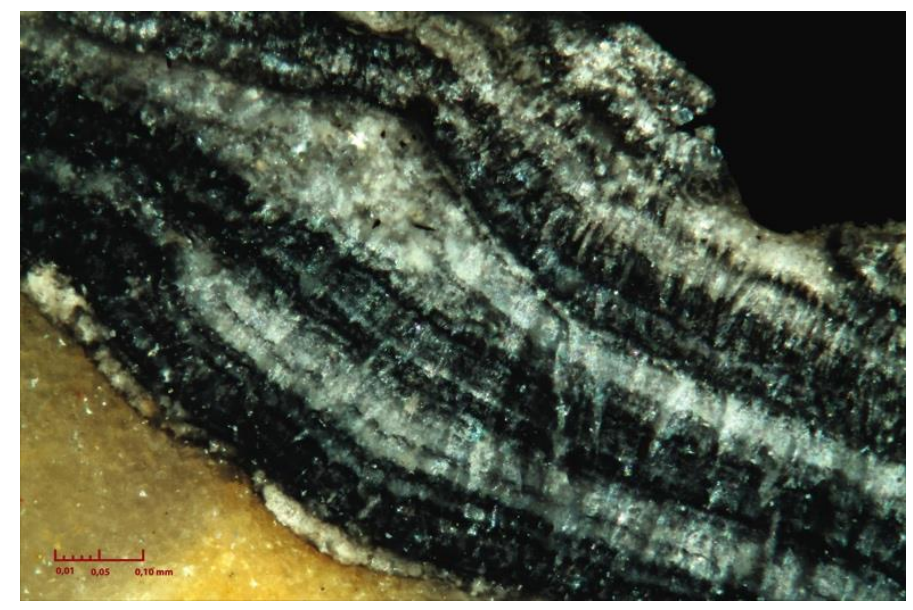

Figure 2. Section polie de l'échantillon l-1217 montrant de multiples dépôts de suie sous forme de très fines lamines noires, Grotte Mandrin, couche I. Microscopie en réflexion (x100), échelle $100 \mu m$.

Les mentions d'inclusion de suie dans des spéléothèmes sont beaucoup plus rares. Et pourtant, tout site en grotte ou en abri-sous-roche où la présence de foyers est attestée présente une forte

\footnotetext{
${ }^{1}$ Les pigments noirs peuvent être, entre autres, le résultat de l'utilisation de charbons ou de noir de suie. Voir notamment la thèse d'Émilie Chalmin (2003) sur la caractérisation du manganèse et l'usage des pigments noirs au Paléolithique.
} 
probabilité d'inclure des dépôts carbonés dans les concrétions pour peu que celles-ci aient été actives. C'est par exemple le cas de la grotte Postojna en Slovénie (Genty et al., 1998; Baker et al., 2008) ou de Salts Cave dans le Kentucky (Benington et al., 1962; Watson \& Yarnell, 1966). À Han-sur-Lesse, en Belgique, il ne s'agit pas de suie mais de charbon et de paille carbonisée provenant des torches utilisées pour l'éclairage lors des visites touristiques modernes (Genty et al., 1997; 1998; Verheyden et al., 2006). D'autres restes charbonneux ont été mis au jour au fond de la grotte de Couffin-Chevaline (Perrette et al., 1997). Nous avons pu recenser à ce jour des traces beaucoup plus anciennes de suie prisonnières de concrétions calcaires dans quelques sites : la Cueva Mayor à Atapuerca en Espagne (Martinez-Pillado et al., 2010 ; 2014), Rana en Norvège (Lauritzen et al., 1990), la grotte Domica en Slovaquie (Petránek \& Pouba, 1951; Aubouin et al., 1996; Gradziòski et al., 2001; Gradziński et al., 2007) et autres grottes slovaques (Kunský, 1939 ; Bárta, 1961 ; Kučera, 1964 : dans Gradzióski et al., 2001), l’Aven de la Portalerie en France (Delannoy et al., 2009), Wierzchowska Górna, Dziewicza, Nietoperzowa, Łokietka, Ciemna, W Straszykowej Górze, Zegar, W Kroczycach et Towarna en Pologne (Gradziński et al., 2003), Jersey cave en Australie (Desmarchelier et al., 2004), la Balma de la Margineda en Andorre (Brochier, 1997 ; 1999 ; 2002 ; 2004 ; 2008) et la Grotte Mandrin en France (Vandevelde et al., 2017 ; 2018).

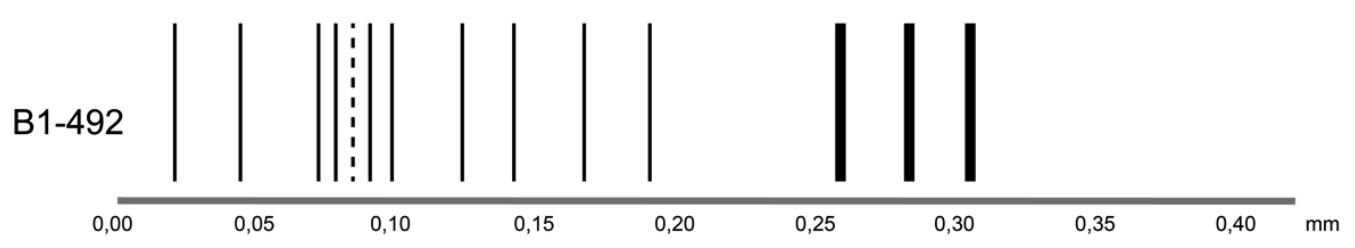

Figure 3. Schéma en " code-barres » des dépôts de suie sur l'échantillon B1-492, Grotte Mandrin, couche

$B$. Les traits continus représentent des films de suie, les traits plus épais représentent des films de suie particulièrement épais et les traits en pointillés symbolisent des dépôts de suie probables. Le trait horizontal représente l'épaisseur totale (en $\mathrm{mm}$ ) de l'encroûtement carbonaté étudié.

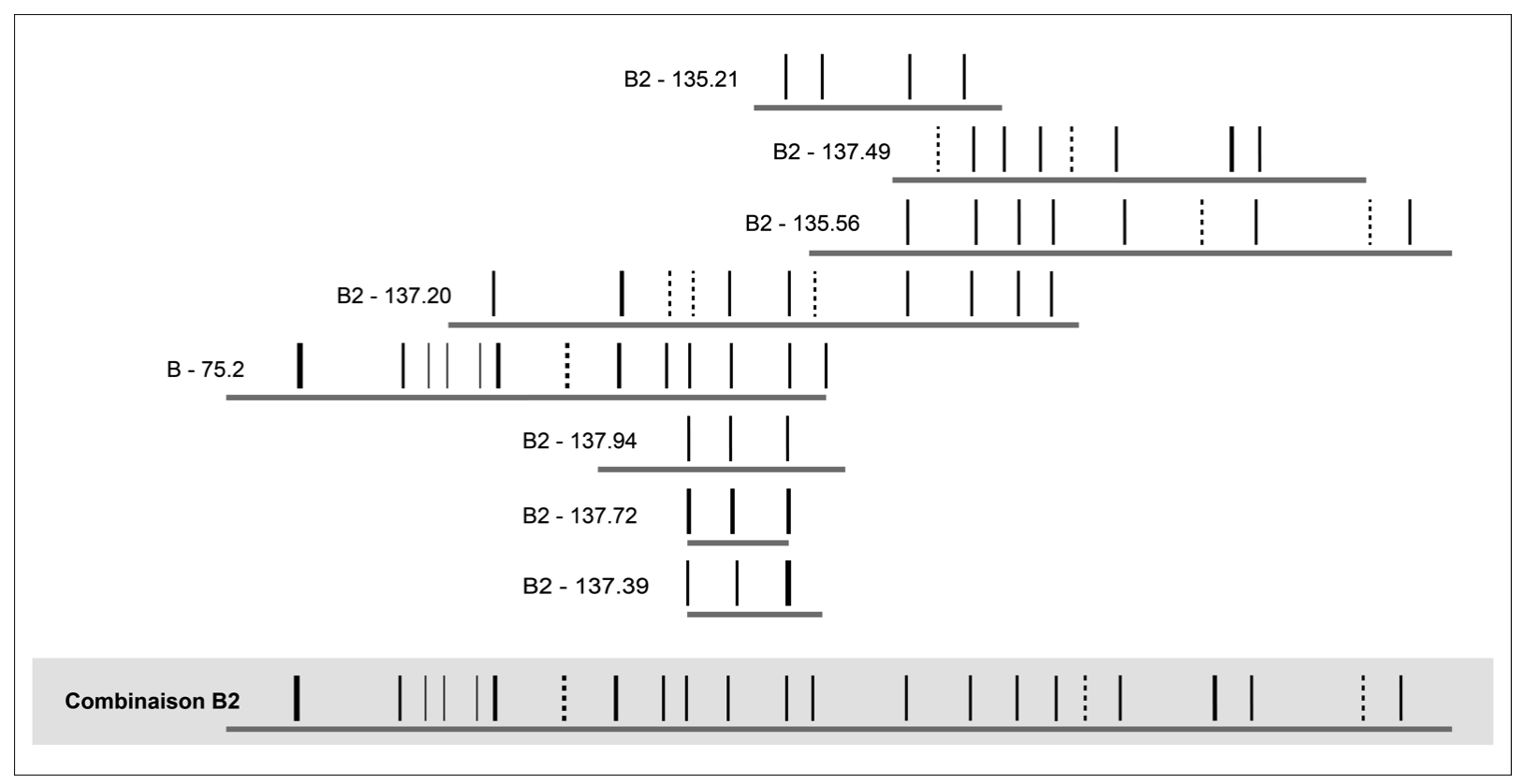

Figure 4. Corrélation des séquences individuelles du niveau B2 et construction de la séquence synthétique des occupations (combinaison B2). Les échantillons B2-137.94, B2-137.72 et B2-137.39 peuvent être placés n'importe où dans la séquence synthétique compte tenu de leur faible nombre de films et de leur espacement relativement régulier. 
Dans ces deux dernières cavités, les encroûtements observés peuvent être considérés comme des constructions stromatolithiques de paroi («calcin de paroi ») recouvrant la surface externe des clastes issus de la dégradation mécanique des parois, qui constituent la majeure partie du remplissage de ces abris. Leur structure stromatolithique bourgeonnante montre la nature biochimique de leur genèse ; elle dépend des précipitations, et, plus généralement, de l'humidité qui conditionne l'activité des organismes vivants, algues, lichens et bactéries. La lamination de ces concrétions est liée aux variations hydrologiques saisonnières, qui conduisent à la formation de lamines micritiques ou micro-sparitiques palissadiques (DCL et WPL ${ }^{2}$ ). Les dépôts noirs peuvent, théoriquement, avoir plusieurs origines. À la Balma de la Margineda comme à la Grotte Mandrin, aucune trace de manganèse n'a pu être mise en évidence dans les encroûtements par l'analyse à la microsonde X. La confusion éventuelle entre une lamine enrichie en oxydes de fer et une lamine de suie peut également facilement être éliminée par une simple observation en lumière réfléchie. De plus, des micro-charbons sont parfois prisonniers des encroûtements à la Grotte Mandrin. Du bois et sans doute aussi des os y ont majoritairement été utilisés comme combustibles dans les nombreux foyers qui se retrouvent tout au long de la stratigraphie. Les lamines noires dans les encroûtements carbonatés sont donc composées de suie, éventuellement de particules charbonneuses, emprisonnées sous des voiles de carbonates de calcium. Autrement dit, les fumées déposent sur les parois des grottes et des abris des films de suie qui, dans des situations favorables, sont conservés dans les encroûtements carbonatés.

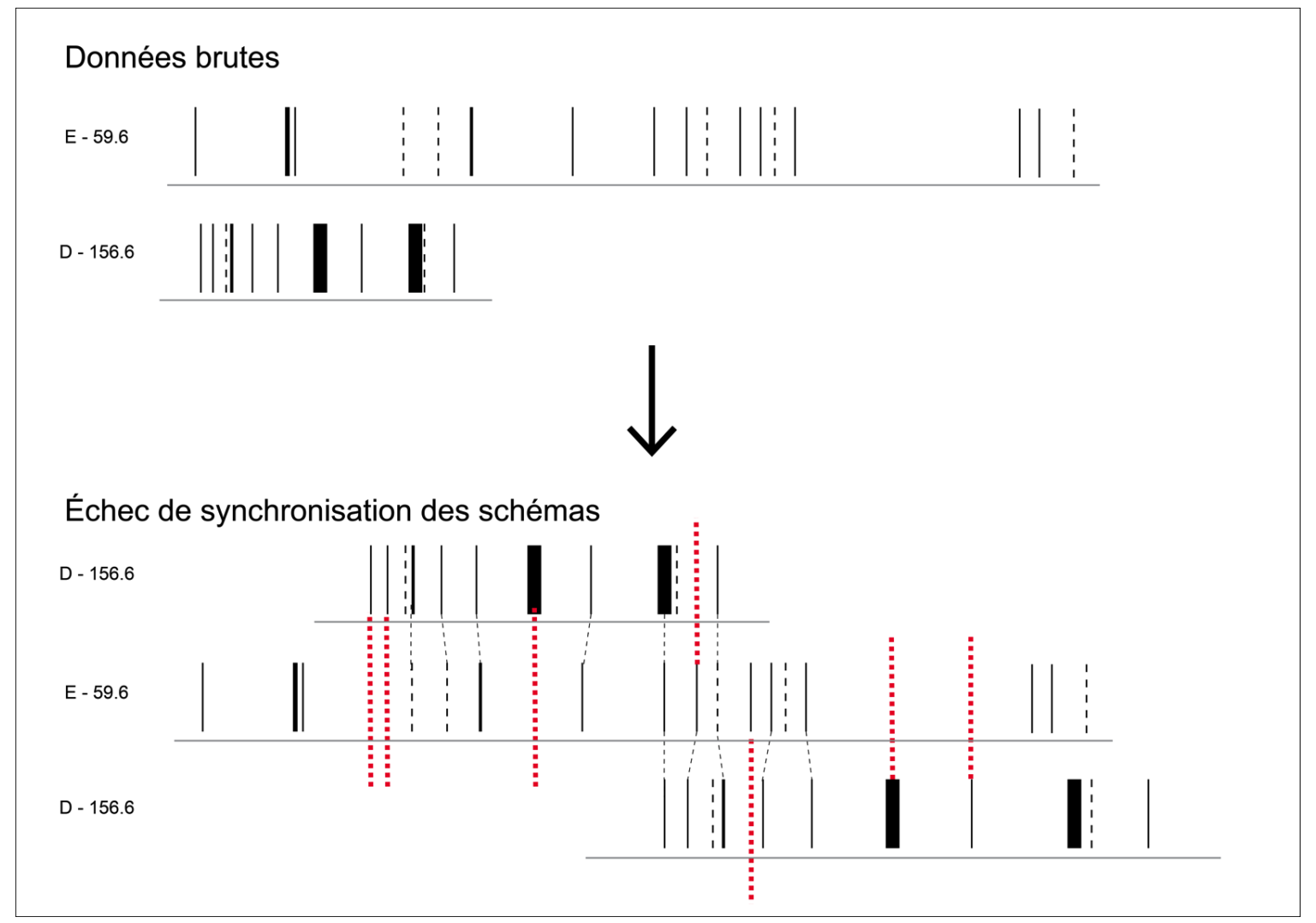

Figure 5. Tentative de synchronisation des séquences de deux échantillons n'appartenant pas à la même unité sédimentaire, Grotte Mandrin, couches $D$ et $E$. Les traits pointillés rouges marquent les conflits de synchronisation.

\footnotetext{
${ }^{2}$ Les DCL désignent les Dark Compact Laminae (sparite palissadique) qui apparaissent sombres en réflexion, alors que les WPL désignent les White Porous Laminae (lamines micritiques) qui sont blanches en réflexion. Le couple DCL-WPL correspond généralement au cycle annuel.
} 


\section{Les encroûtements carbonés de paroi : un outil micro-chronologique}

Jusqu'à présent, les quelques études qui ont été réalisées sur les lamines noires prisonnières des encroûtements ont toutes conclu que ces dépôts de suie constituaient de très bons marqueurs de la présence humaine. Ils ont parfois pu être directement datés afin d'identifier les périodes de fréquentation d'un site (p. ex. à la grotte Domica, Pétránek \& Pouba, 1951; Gradziòski et al., 2001; Gradziński et al., 2003 ; 2007). Le caractère saisonnier des occupations (p. ex. Genty et al., 1997 ; Verheyden et al., 2006; Martínez-Pillado et al., 2010; 2014) a également pu être précisé à partir de la position des films dans les doublets DCL-WPL. Nous avons pu remarquer que l'étude de ces dépôts carbonés n'est pas systématique et que si les questions de micro-chronologie des occupations et de lien entre dépôt archéologique et spéléothèmes sont abordées, elles ne le sont jamais conjointement. À l'exception du cas de la Balma de la Margineda présenté plus loin, aucune étude n'a porté sur la construction de chroniques micro-chronologiques de la fréquentation humaine d'une cavité en lien avec la constitution des dépôts archéologiques.

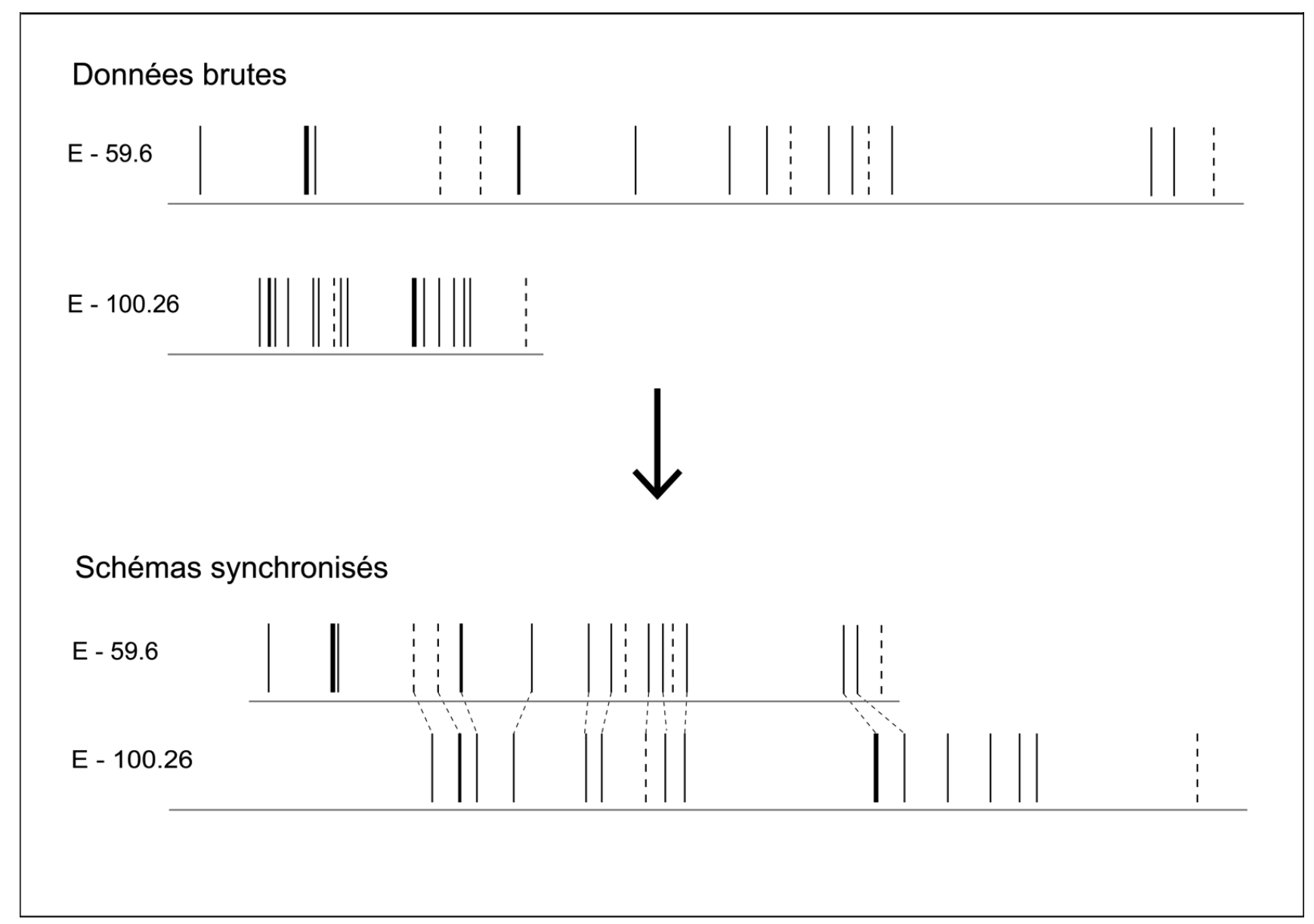

Figure 6. Synchronisation des séquences de deux échantillons appartenant à la même unité sédimentaire, Grotte Mandrin, couche E.

Nous avons observé des dépôts de suie d'âge tardiglaciaire à la Balma de la Margineda et du Pléistocène supérieur à la Grotte Mandrin, cimentés dans des encroûtements carbonatés présents à la surface des clastes et collectés dans les différentes unités sédimentaires de ces deux abris. L'observation microscopique de lames minces et/ou de sections polies réalisées dans l'axe de croissance des concrétions montre que ce qui pouvait apparaître comme une unique lamine noire à l'œil nu ou à faible grossissement (figure 1) correspond généralement en une multitude de films, fins de quelques micromètres d'épaisseur (figure 2). Ces séries de films de suie sont autant de témoins 
des nombreuses occupations des hommes dans la cavité. Ils sont documentés graphiquement, à partir de photographies, sous forme de schémas en «code-barres » (figure 3), sur lesquels les traits continus noirs représentent les films de suie et l'espace qui les sépare correspond aux lamines carbonatées cristallisées en l'absence de fréquentation humaine. Dans chaque unité sédimentaire, les différents échantillons analysés permettent, chacun, de construire une séquence. En corrélant ces différents «codes-barres » relevés sur les fragments provenant d'une même unité sédimentaire, il est possible d'établir une séquence synthétique (figure 4) qui documente l'ensemble des fréquentations humaines correspondantes. Le décompte des films de suie par unité sédimentaire permet ainsi d'obtenir un Nombre Minimum d'Occupations (NMO). Si l'on constate qu'il est généralement impossible de faire coïncider les séquences relevées sur des fragments provenant de deux couches différentes par un jeu de dilatation/contraction des schémas (figure 5), il est par contre possible de le faire entre échantillons d'une même couche (figure 6). Ceci démontre, a posteriori, que les vitesses de croissance des encroûtements, bien que différentes en chaque point de la paroi, suivent les mêmes évolutions. La micro-chronologie des occupations successives des grottes et des abris-sousroche, incluant les notions de phases d'occupation ${ }^{3}$ et de rythmicité des occupations, devient alors accessible avec une résolution qui, dans les cas les plus favorables, peut être annuelle.
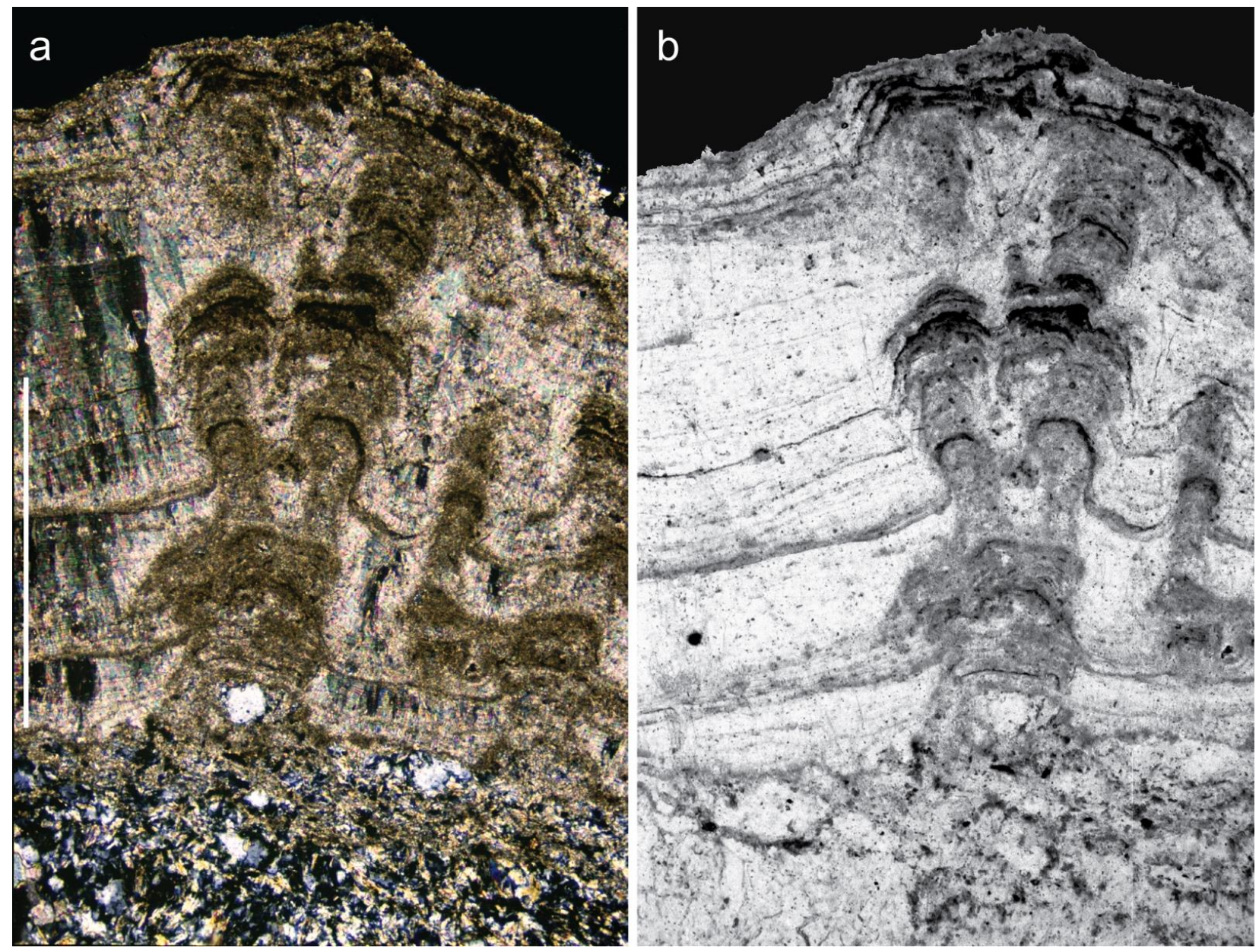

Figure 7. Encroûtement carbonaté pariétal contenant des films de suie, Balma de la Margineda, couche 7. Échelle $500 \mu \mathrm{m}$. Les films de suie sont préférentiellement conservés dans les micro-colonnes. a : lumière polarisée analysée ; $b$ : lumière polarisée, diaphragme d'ouverture ouvert, afin de mettre en évidence les matériaux opaques, ici les films de suie.

\footnotetext{
${ }^{3}$ Les phases d'occupation sont des ensembles d'occupations séparés par de longs hiatus ou différenciés par des rythmicités d'occupation différentes.
} 


\section{1. À l'origine de la méthode. Le cas de la Balma de la Margineda}

À notre connaissance, la première application à des problèmes archéologiques a été réalisée à partir de minces fragments de stromatolithes pariétaux sur plaquettes schisteuses retrouvés dispersés dans les dépôts tardiglaciaires d'un abri-sous-roche pyrénéen d'altitude, la Balma de la Margineda (Guilaine \& Martzluff, 1995 ; Brochier, 1997 ; 1999 ; 2002 ; 2008 ; Guilaine et al., 2008). Ces fragments sont fins, rares et très petits, toujours largement inférieurs au centimètre carré. Ils justifient, à eux seuls, l'observation méticuleuse de tous les éléments grossiers extraits d'échantillons sédimentaires volumineux. L'observation en lames minces montre que ces encroûtements sont formés de l'alternance de lamines micritiques et de lamines sparitiques palissadiques (figures $7 a$ et 8 ). Celles-ci forment par endroits des dômes d'aspect stromatolithique, sites sur lesquels ont été préférentiellement piégés - ou conservés - les dépôts carbonés (figure 7b). Dans cette succession de nombreux doublets DCL-WPL, seules les lamines micritiques portent des films de suie. Les fragments récoltés dans les quelques unités archéologiques aziliennes successives, les couches 9 à 7 , présentent une telle similitude dans les rythmes d'occupation (absence ou présence de film de suie) qu'il faut conclure à une seule période séculaire de concrétionnement, que cette période représente une partie (ou la totalité) du temps correspondant à une seule couche archéologique, la couche 9 (figure 9).

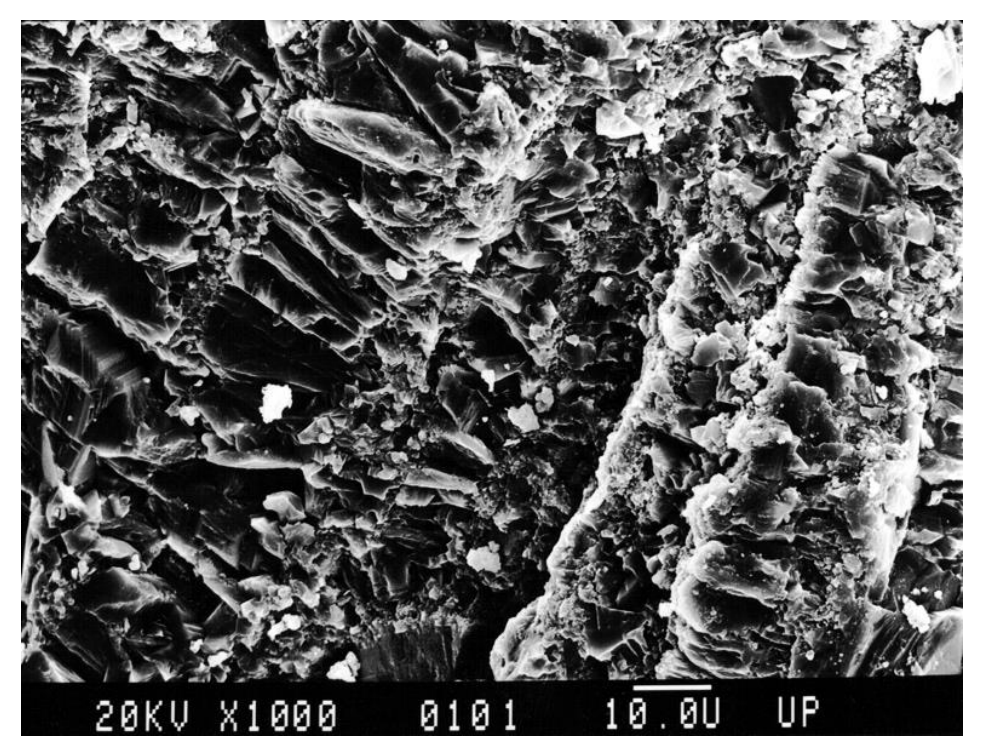

Figure 8. Alternance de lamines micritiques et sparitiques dans un encroûtement pariétal de la Balma de la Margineda. Microscopie électronique à balayage, échelle $10 \mu \mathrm{m}$.

Les caractéristiques climatiques locales actuelles et les processus hydrologiques associés, largement conditionnés par le relief et l'enneigement, ne font que conforter le caractère annuel des doublets. La couche archéologique azilienne (C9) apparaît ainsi résulter du cumul (et non pas être un palimpseste !) de très nombreuses $(\mathrm{n}>30)$ occupations annuelles, parfois consécutives, toujours à la même période de l'année. Au-delà de l'information archéologique : l'abri n'était occupé que quelques mois dans l'année, régulièrement, toujours à la même saison (à l'époque du rut des bouquetins) par un groupe de chasseurs (-collecteurs); il était donc intégré dans un parcours le reliant à d'autres sites ; l'information fondamentale est certainement celle qui oblige à considérer la couche archéologique comme un objet composite et cumulatif. En d'autres termes, le synchronisme des différents témoins recueillis dans cette couche azilienne unique (industrie, faune... et structures) est bien loin d'être assuré. 


\subsection{Adaptation au cas de la Grotte Mandrin}

La Grotte Mandrin est un abri-sous-roche situé dans la moyenne vallée du Rhône, sur la rive gauche du fleuve, une dizaine de kilomètres au sud de Montélimar. Les fouilles ont commencé en 1991 avec les travaux d'Yves Giraud et se poursuivent actuellement sous la direction de Ludovic Slimak. Le premier niveau (A) s'est mis en place entre la fin du Néolithique (dépôt funéraire collectif à crémations répétées) et la période moderne ; les niveaux sous-jacents (B à G) peuvent être divisés en six phases culturelles associées aux derniers Néandertaliens et aux premiers Homo sapiens régionaux : le niveau $\mathrm{B} 1$, daté autour de $42 \mathrm{ka}$ cal $\mathrm{BP}$, est attribué au Protoaurignacien; viennent ensuite le Post-Néronien II (niveaux B2, B3, B/C, C1 et C2), le Post-Néronien I (niveau D), le Néronien (niveau E), le Moustérien de type Quina rhodanien (couche F) et le Moustérien de type Ferrassie (couche G) ; ces phases couvrent la fin du Paléolithique moyen, d'environ 42 à 5055 ka cal BP (Slimak, 2004 ; Higham et al., 2014, SOM : 34-38). Les niveaux H, I et J sont également attribués au Moustérien.

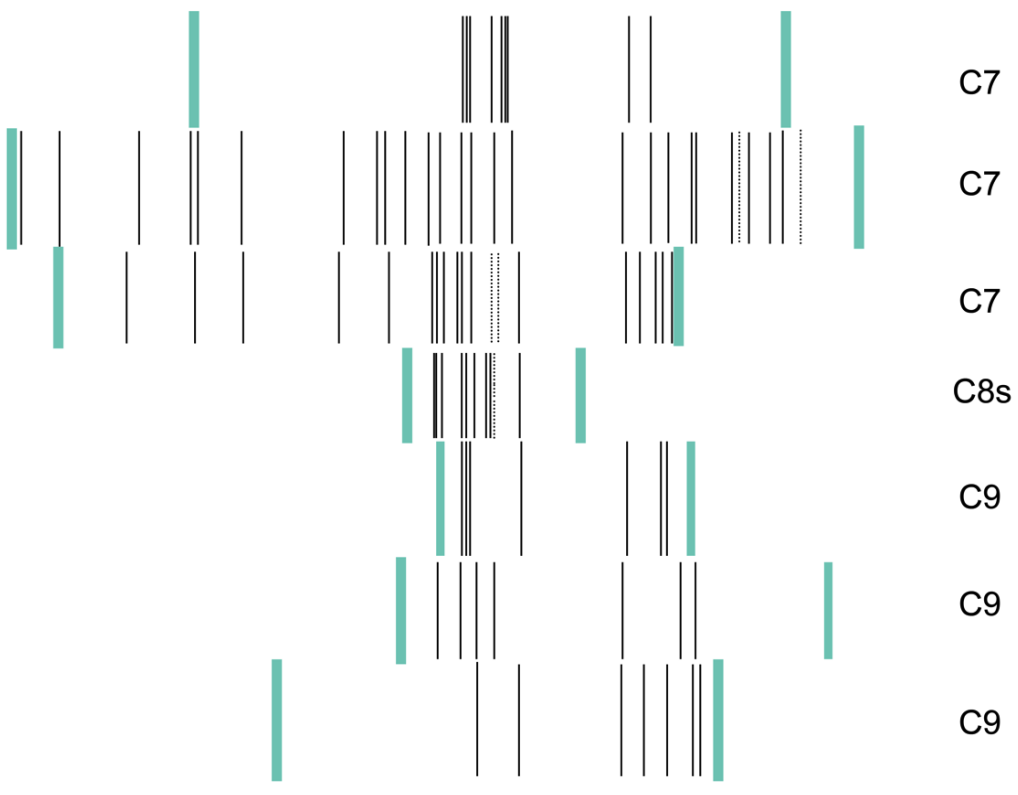

Figure 9. Synchronisation des séquences individuelles obtenues dans les différentes unités sédimentaires de la Balma de la Margineda. Les traits épais encadrant les "codes-barres " représentent le début et la fin des encroûtements carbonatés contenant les dépôts de suie.

Une adaptation de la méthode micro-chronologique présentée plus haut y a été réalisée. De la même manière qu'à la Balma de la Margineda, il s'agissait d'établir une micro-chronologie des occupations humaines, à partir des dépôts de suie pariétaux en utilisant, dans ce cas, un grand nombre d'échantillons et donc, dans la mesure du possible, sans avoir recours à une technique aussi lourde que celle de l'analyse à partir de lames minces. L'observation de surfaces polies a été choisie. En moyenne, une dizaine de plaquettes calcaires portant un encroûtement carboné fut sélectionnée par choix aléatoire dans chaque niveau archéologique pour cette étude en série. Le protocole de préparation, d'observation et d'analyse des encroûtements qui s'est révélé le plus adapté est le suivant :

- Sciage et polissage ${ }^{4}$ de la section de la plaquette calcaire encroûtée

\footnotetext{
${ }^{4}$ Après de nombreux essais, les grains les plus adaptés à l'observation des films de suie sont les grains 500 FEPA (12,8 $\mu$ m) et 600 FEPA $(9,3 \mu \mathrm{m})$. Si le grain est trop fin, cela provoque une sorte d'étalement de la suie, avec pour conséquence une mauvaise 
- Observation au microscope en réflexion et décompte du nombre de films

- Photographies de la zone où les films sont les plus nombreux et les plus visibles

- Mesure des distances entre les films et schématisation des données dans le style graphique d'un « code-barres »

- Vérification des schémas produits sous le microscope

- Dilatation/contraction graphique des schémas afin de trouver des corrélations entre eux et de construire une séquence synthétique de films par niveau archéologique.

\subsection{Résultats et implications}

D'après des observations actualistes à la Grotte Mandrin, la suie peut être recouverte par un voile calcaire en quelques mois (de l'ordre de la saison) ou bien ne jamais l'être, puisque la vitesse de croissance du concrétionnement varie en fonction de l'état hydrique aux différents points de la voûte; il existe donc des secteurs de la paroi où la formation d'encroûtements n'a pas lieu. La desquamation ne se fait pas non plus partout au même moment; les plaquettes portent donc à leur surface des enduits carbonés composés de séquences différentes. Il est logique de proposer que ces différentes séquences sont corrélables entre elles puisqu'elles sont les témoins des mêmes événements. À la manière de la dendrochronologie, les séquences les plus anciennes, détachées en premier de la paroi, recoupent des séquences enregistrées par des fragments détachés plus tardivement. De même, certaines plaquettes ont enregistré, avant leur détachement, une longue séquence de films de suie; d'autres, plus courtes, se révèlent souvent n'être que des extraits de séquences plus longues.

\begin{tabular}{|cccc|}
\hline Couche & Niveau & Période & NMO \\
\hline A & & Néolithique & 29 \\
\hline & B1 & Protoaurignacien & 6 \\
\cline { 2 - 4 } B & B2 & Moustérien (Post-Néronien II) & 9 \\
& B3 & Moustérien (Post-Néronien II) & 14 \\
\hline C & C1 & Moustérien (Post-Néronien II) & 38 \\
\hline D & C2 & Moustérien (Post-Néronien II) & 30 \\
\hline E & & Moustérien (Post-Néronien I) & 30 \\
\hline F & & Néronien & 18 \\
\hline G & & Moustérien (Quina Rhodanien) & 21 \\
\hline H & & Moustérien & 12 \\
\hline I & & Moustérien & 8 \\
\hline J & & Moustérien & 70 \\
\hline Total & A à J & Moustérien & 18 \\
\hline
\end{tabular}

Tableau 1. Nombre Minimum d'Occupations (NMO) par niveau archéologique à la Grotte Mandrin. État de la recherche en 2015.

distinction des différents films de suie. Pour améliorer la lisibilité des échantillons, l’ajout de vernis sur les surfaces observées est judicieux. 
Si l'on espère apporter des éléments micro-chronologiques pour comprendre la succession d'occupations dans chaque ensemble sédimentaire et établir un NMO par couche, il est essentiel de savoir si les fragments de paroi se délitent assez vite pour être contemporains de la couche dans laquelle ils tombent. Une séquence longue d'une trentaine de films de suie a pu être reconstituée à la Balma de la Margineda (Andorre), en synchronisant les différentes séquences individuelles. Les plaquettes schisteuses portant des encroûtements carbonés à leur surface sont présentes dans toutes les unités sédimentaires de cet abri mais les séquences individuelles peuvent être aisément synchronisées, ce qui implique que les encroûtements se sont formés durant une même période séculaire, à l'Azilien, et que la paroi s'est ensuite lentement délitée dans les niveaux postérieurs. À l'inverse, à la Grotte Mandrin, les séquences individuelles ne se recoupent qu'au sein d'une même unité sédimentaire (figures 5 et 6). Cela signifie que la paroi se délitait suffisamment rapidement pour que la formation des calcins carbonés soit globalement contemporaine des niveaux dans lesquels ils sont retrouvés (Vandevelde et al., 2017; 2018). Puisqu'une plaquette retrouvée en E peut porter à sa surface la séquence des occupations des niveaux antérieurs (p. ex. F) et contemporains (E), mais pas postérieurs (p. ex. D), il a été possible d'établir, non pas une séquence séculaire d'occupation de l'abri, mais des chroniques d'occupations correspondant au dépôt de chaque unité sédimentaire. Chaque niveau présente un rythme d'occupations qui lui est propre: des phases d'occupations sont parfois identifiables et il est possible de quantifier précisément le nombre minimum d'occupations dans l'abri, par niveau archéologique et pour l'ensemble de la stratigraphie. Même si on ne contrôle pas précisément le temps à partir de ces encroûtements carbonatés, comme cela pourrait être le cas à partir de spéléothèmes, il a été possible d'obtenir à Mandrin des NMO par couche: 13 niveaux archéologiques se transforment en un minimum de 273 occupations (tableau 1), dont au moins 243 pour les 12 niveaux paléolithiques. Une telle résolution microchronologique est fondamentale pour l'archéologue puisqu'elle a, par exemple, de lourdes conséquences sur l'interprétation des études spatiales.

Il en ressort une perception nouvelle de «l'occupation». Un «sol d'occupation » tel que défini par Bordes (1975) et Binford (1982), est «une surface reconnaissable sur laquelle a vécu l'homme paléolithique pendant un laps de temps suffisamment court pour qu'on puisse espérer déduire de la position des vestiges quelque chose au sujet de ses activités »(Bordes, 1975: 139); c'est le résultat d'une seule occupation de courte durée, pouvant être définie comme un usage ininterrompu d'un lieu par les membres participant à un système culturel (Binford, 1982); il s'agirait donc en réalité d'un objet purement théorique. Bordes (1975) avait déjà souligné le fait que les couches archéologiques sont des unités cumulatives. À Mandrin, même dans le cas où un «sol » est reconnaissable par la dispersion horizontale non homogène du matériel et une identification de structures anthropiques (foyer et structure circulaire de blocs calcaires et ossements - «cabane ») dans la couche E, il s'avère que de multiples occupations $(\mathrm{NMO}=18)$ s'y sont néanmoins succédées. Si certaines études micromorphologiques permettent de mettre en évidence différents niveaux d'occupation au sein d'une même couche (voir par exemple Boivin, 2000), il est naturellement impossible de les isoler à la fouille (Brochier, 1999; Aldeias et al., 2012). Au vu des études micro-chronologiques réalisées sur les encroûtements carbonatés à la Balma de la Margineda et à la Grotte Mandrin, il semble évident que ce qui est observé archéologiquement dans le cas d'un «sol» n'est pas l'objet théorique défini plus haut mais plutôt une surface reconnaissable, sur laquelle a vécu l'homme paléolithique, où l'on peut espérer déduire de la position des vestiges quelque chose au sujet de ses activités et qui livre les témoins intimement associés résultant de plusieurs occupations. Celle-ci peut correspondre à la fréquentation répétée d'un lieu, possiblement toujours à la même saison et/ou pour la même activité, par les membres d'un même groupe culturel et dont l'utilisation de l'espace reste globalement la même à chaque occupation. 
Les couches archéologiques et les «sols» doivent donc être considérés comme des unités cumulatives, témoignant de multiples occupations. Nous aurions pu tout aussi bien parler de «palimpsestes », puisque ce terme, qui désigne à l'origine un "parchemin dont la première écriture, grattée ou lavée, a fait place à un nouveau texte», est largement utilisé dans la littérature archéologique. Dans le domaine archéologique, ce concept renvoie à de nombreuses définitions : il peut tout aussi bien faire référence, dans sa forme la plus stricte et littérale, aux phénomènes où toutes traces d'activités anciennes ont disparu en dehors des plus récentes, qu'au palimpseste dit «cumulatif», où toutes les traces sont conservées mais tellement remaniées qu'elles ne sont pas séparables en leurs composantes originelles. Notons qu'il existe également les palimpsestes «spatiaux», «temporels» ou «de signification» (Bailey, 2007: 204-208). Il apparaît évident, considérant les nombreuses connotations qui l'accompagnent, que l'usage de ce vocable manque singulièrement de clarté, voire d'utilité. Ce qui reste une puissante métaphore en littérature se mue en un concept trop peu explicite et pluriel, dont il vaudrait mieux se passer dans les disciplines (géo)archéologiques au profit de termes moins consensuels et plus précis.

\section{Perspectives}

Une application évidente des multiples potentiels d'étude de ces dépôts carbonés dans les encroûtements carbonatés concerne le problème de l'origine de l'utilisation du feu : puisque le recours au feu dans un abri ou une grotte peut laisser des traces dans les concrétions pariétales, la présence de plusieurs films de suie dans un même encroûtement carbonaté peut constituer un argument fort de l'utilisation répétée, et donc maîtrisée, de cette technique ${ }^{5}$. Il est en outre possible de dater directement la suie pour les périodes récentes ou de dater directement l'encroûtement pour les périodes plus anciennes.

Des analyses de chimie organique des dépôts de suie sont actuellement envisagées. L'odeur de graisse brûlée ou de goudron est toujours présente dans le cas de la Grotte Mandrin, et ressort très nettement lorsque l'on gratte ces enduits carbonés. Cela signifie que la matière organique a été préservée dans la calcite et que son analyse pourrait nous permettre d'en préciser la nature.

L'analyse d'un plus grand nombre d'échantillons est nécessaire pour retracer la séquence complète des occupations sur la totalité des niveaux mis au jour (A-J) à la Grotte Mandrin. Les prochaines analyses seront donc préférentiellement axées sur les échantillons relevés aux interfaces entre les couches et sur l'augmentation du nombre de plaquettes étudiées pour chaque niveau archéologique. Les liens B2-B1 et F-E-D seront, bien sûr, le sujet de toutes les attentions, au vu des enjeux archéologiques qui s'y rapportent. Préciser la micro-chronologie des dernières occupations moustériennes du niveau B2 (Post-Néronien II), probablement par les Néandertaliens, en lien avec celles du niveau B1, potentiellement associé à Homo sapiens (Protoaurignacien), serait une avancée majeure dans la compréhension de la transition Paléolithique moyen/Paléolithique supérieur. De même, le Néronien (couche $\mathrm{E}$ ) est techniquement très moderne et très différent à la fois du niveau Quina sous-jacent (couche F) et du Post-Néronien I (couche D) qui lui succède. C'est un niveau charnière dont les relations micro-chronologiques avec le Moustérien Quina et le Post-Néronien (sous et sus-jacents) sont de première importance. Le perfectionnement de la méthode, surtout en ce qui concerne le traitement, l'analyse des images et la traduction des données brutes en schéma via le

\footnotetext{
${ }^{5}$ Des incendies récurrents peuvent également laisser leur trace dans les spéléothèmes (Desmarchelier et al., 2004). C'est alors la présence humaine sur le site, la répétition, la fréquence et la localisation des films dans les doublets DCL-WPL qui permettent de juger de la nature anthropique ou non des feux. En effet, on imagine mal une répétition de feux naturels lors des saisons d'excès hydriques; au contraire, c'est un scénario plausible lors des saisons sèches. Si les films de suie ne sont pas localisés uniquement dans les WPL, le caractère anthropique des feux ayant laissé les traces de suie est donc hautement probable.
} 
module LnSeq de la plateforme Datawald (GL Conception, 2015) est en cours. Le traitement statistique des séquences devrait également permettre de préciser la qualité des synchronismes.

Au-delà de l'établissement d'un NMO, la rythmicité des dépôts de films de suie est à étudier. Elle peut être saisonnière, comme à la Balma de la Margineda, mais ce n'est pas nécessairement la règle. De la même manière que Boivin (2000) démontre la cyclicité des enduits de sols et de murs par des comparaisons ethnographiques, les études sur la mobilité de groupes humains actuels et sur leur occupation des abris-sous-roche ou des grottes dans un territoire devront être réalisées afin de créer un cadre de référence actualiste, puisqu'il se dessine, avec l'étude des rythmes, un potentiel archéologique.

Les perspectives de recherche sur les dépôts de suie sont nombreuses et prometteuses. Il s'agit d'un outil micro-chronologique performant permettant de répondre à des questions archéologiques fondamentales. La méthode d'analyse mise en place ne demande qu'à être perfectionnée par l'intégration de nouveaux outils. Il semblerait que de nombreux sites recèlent ce type de vestiges et, au vu des résultats obtenus à la Balma de la Margineda et à la Grotte Mandrin, il se pourrait que ces dépôts de suie ouvrent une nouvelle voie à la compréhension des sociétés humaines passées.

REMARQUE. Cet article a été rédigé dans le cadre du colloque «Journées d'étude géoarchéologiques : Dépôts carbonatés en contexte archéologique, une mémoire de la gestion de l'eau » organisé par C. Petit et J. Curie en septembre 2014. Il présente notamment les résultats de Master de S. Vandevelde, qui sont préliminaires en ce qui concerne les Nombres Minimums d'Occupations documentés à la Grotte Mandrin.

\section{Remerciements}

Nous tenons à remercier les organisateurs des Journées d'étude géoarchéologiques pour leur invitation à venir présenter nos travaux. Nous remercions également le Service Régional de l'Archéologie Auvergne-Rhône-Alpes et la ville de Malataverne (Drôme), qui financent les recherches à la Grotte Mandrin.

\section{Conflit d'intérêts}

Aucun conflit d'intérêts à déclarer.

\section{Évaluation}

Les deux rapporteurs de cet article sont Christophe Petit et Julien Curie.

\section{Responsabilités des évaluateurs externes}

Les évaluations des examinateurs externes sont prises en considération de façon sérieuse par les éditeurs et les auteurs dans la préparation des manuscrits pour publication. Toutefois, être nommé comme examinateur n'indique pas nécessairement l'approbation de ce manuscrit. Les éditeurs d'Archéologie, Société, Environnement assument la responsabilité entière de l'acceptation finale et la publication d'un article.

\section{Références bibliographiques}

Aldeias, V., Goldberg, P., Sandgathe, D., Berna, F., Dibble, H.L., McPherron, S.P., Turq, A., Rezek, Z., 2012. Evidence for Neandertal use of fire at Roc de Marsal (France). Journal of Archaeological Science. 39, $2414-2423$. 
Aubouin, J., Brousse, R., Lehman, J.-P., 1996. Précis de géologie, t. 1 : Pétrologie. Dunod, Paris.

Bailey, G., 2007. Time perspectives, palimpsests and the archaeology of time. Journal of Anthropological Archaeology. 26, 198-223.

Baker, A., Smith, C.L., Jex, C., Fairchild, I.J., Genty, D., Fuller, L., 2008. Annually laminated speleothems: a review. International Journal of Speleology. 37, 193-206.

Benington, F., Melton, C., Watson, P.J., 1962. Carbon Dating Prehistoric Soot from Salts Cave, Kentucky. American Antiquity. 28, 238-241.

Binford, L.R., 1982. The archaeology of place. Journal of Anthropological Archaeology. 1, 5-31.

Boivin, N., 2000. Life rhythms and floor sequences: excavating time in rural Rajasthan and Neolithic Catalhoyuk. World Archaeology. 31, 367-388.

Bordes, F., 1975. Sur la notion de sol d'habitat en préhistoire paléolithique. Bulletin de la Société préhistorique française. Comptes rendus des séances mensuelles. 72, 139-144.

Brochier, J.É., 1997. Couches archéologiques ou «sols d'habitat»? Quelques observations micro-chronologiques dans un abri-sous-roche pyrénéen. Presented at the International Workshop on Archaeological Soil Micromorphology, Basel.

Brochier, J.É., 1999. Couche archéologique, sol archéologique et distributions spatiales: quelques réflexions (géo)archéologiques sur un vieux problème. Geoarqueologia i Quaternari litoral. Memorial Maria Pilar Fumanal, 91-95.

Brochier, J.É., 2002. Les sédiments anthropiques: Méthodes d'étude et perspectives, in: Miskovsky, J.-C. (Éd.), Géologie de La Préhistoire: Méthodes, Techniques, Applications. Presses universitaires de Perpignan, Paris, pp. 453-477.

Brochier, J.É., 2004. De la sédimentologie à la géoarchéologie. Un demi-siècle de recherches françaises dans les entrées de grottes et les abris-sous-roche, in: Évin, J. (Éd.), presented at the $\mathrm{XXVI}^{\mathrm{e}}$ congrès préhistorique de France. Congrès du centenaire: Un siècle de construction du discours scientifique en Préhistoire, Société préhistorique française, Paris, pp. 31-46.

Brochier, J.É., 2008. Estudi geoarqueològic dels dipòsits tardiglacials de la Balma de la Margineda (Capes de la 6 a la 11), in: Guilaine, J., Martzluff, M. (Éds.), Les Excavacions a La Balma de La Margineda (1979-1991). Minister d'Afers socials i Cultura, Andorra, pp. 52-60.

Clottes, J., Chauvet, J.-M., Brunel-Deschamps, E., Hillaire, C., Daugas, J.-P., Arnold, M., Cachier, H., Evin, J., Fortin, P., Oberlin, C., 1995. Les peintures paléolithiques de la Grotte Chauvet-Pont d'Arc, à Vallon-Pont-d'Arc (Ardèche, France): datations directes et indirectes par la méthode du radiocarbone. Comptes rendus de l'Académie des sciences. Série 2. Sciences de la terre et des planètes. 320, 1133-1140.

Delannoy, J.-J., Gauchon, C., Hobléa, F., Jaillet, S., Maire, R., Perrette, Y., Perroux, A.-S., Ployon, E., Vanara, N., 2009. Karst: from palaeogeographic archives to environmental indicators. Géomorphologie: relief, processus, environnement. 2, 83-94.

Desmarchelier, J.M., Hellstrom, J., Spate, A., 2004. Constraining relative wildfire frequency in the Australian alps over the past 500,000 years, using U- Th dating of speleothem-encapsulated soot layers. Presented at the Biennial Conference of the Australiasian Quaternary Association, p. 19.

Duncan, S., 1997. Examining Early Nineteenth century Saltpeter Caves: an Archaeological Perspective. Journal of Cave and Karst Studies. 59, 91-94.

Genty, D., Dauphin, Y., Deflandre, G., Quinif, Y., 1997. Exemples de particules d'origine anthropique piégées dans les lamines de croissance de stalagmites - Intérêt pour la reconstitution des environnements humains anciens [Examples of anthropogenic particles trapped into stalagmite growth laminae - Interest for the study of prehistoric human activity.]. Quaternaire. 8, 149-157.

Genty, D., Vokal, B., Obelic, B., Massault, M., 1998. Bomb 14C time history recorded in two modern stalagmites importance for soil organic matter dynamics and bomb 14C distribution over continents. Earth and Planetary Science Letters. 160, 795-809.

G.L. Conception, 2015. DataWald - Module LnSeq. 
González, M.E.I., Martínez, P.F., 1998. Hallazgos rupestres en torno a la antigua ruta de Zeugma a Edessa, in: Romanización y Cristianismo En La Siria Mesopotámica. pp. 119-127.

Gradziński, M., Górny, A., Pazdur, A., Pazdur, M.F., 2003. Origin of black coloured laminae in speleothems from the Kraków-Wieluń Upland, Poland. Boreas. 32, 532-542.

Gradziński, M., Hercman, H., Nowak, M., Bella, P., 2007. Age of Black Coloured Laminae Within Speleothems from Domica Cave and Its Significance for Dating of Prehistoric Human Settlement. Geochronometria. 28, 39-45.

Gradziòski, M., Hercman, H., Nowicki, T., Bella, P., 2001. Dark Coloured Laminae Within Speleothems as an Indicator of the Prehistoric Man Activity: Case Study from Domica Cave (Slovakia), Preliminary Results. Presented at the 13th International Congress of Speleology, 4th Speleological Congress of Latin América and Caribbean, 26th Brazilian Congress of Speleology, Brasília DF, pp. 263-267.

Guilaine, J., Barbaza, M., Martzluff, M., 2008. Les excavacions a la Balma de la Margineda (1979-1991). Edicions del Govern d'Andorra.

Guilaine, J., Martzluff, M., 1995. Les excavacions a la Balma de la Margineda (1979-1991). Edicions del Govern d'Andorra.

Higham, T., Douka, K., Wood, R., Ramsey, C.B., Brock, F., Basell, L., Camps, M., Arrizabalaga, A., Baena, J., Barroso-Ruiz, C., Bergman, C., Boitard, C., Boscato, P., Caparros, M., Conard, N.J., Draily, C., Froment, A., Galvan, B., Gambassini, P., Garcia-Moreno, A., Grimaldi, S., Haesaerts, P., Holt, B., Iriarte-Chiapusso, M.-J., Jelinek, A., Jorda Pardo, J.F., Maillo-Fernandez, J.-M., Marom, A., Maroto, J., Menendez, M., Metz, L., Morin, E., Moroni, A., Negrino, F., Panagopoulou, E., Peresani, M., Pirson, S., de la Rasilla, M., Riel-Salvatore, J., Ronchitelli, A., Santamaria, D., Semal, P., Slimak, L., Soler, J., Soler, N., Villaluenga, A., Pinhasi, R., Jacobi, R., 2014. The timing and spatiotemporal patterning of Neanderthal disappearance. Nature. 512, 306-309 and Supplementary information.

Ishihara-Brito, R., Guerra, J., 2012. Chapter 4: Windows of the Earth: An Ethnoarchaeological Study on Cave Use in Suchitepéquez and Sololá, Guatemala, in: AMCS Bulletin 23. Heart of Earth: Studies in Maya Ritual Cave Use. pp. 51-60.

Lachenal, T., Rucker, C., 2009. L'aven de la Mort de Lambert (Valbonne, Alpes-Maritimes) et les dépôts de vases en grotte à l'âge du Bronze en France méridionale, in : Bonnardin, S., Hamon, C., Lauwers, M., Quilliec, B. (Éds.), presented at the $\mathrm{XXIX}^{\mathrm{e}}$ rencontres internationales d'archéologie et d'histoire d'Antibes. Du matériel au spirituel : réalités archéologiques et historiques des «dépôts » de la Préhistoire à nos jours, Éditions APDCA, pp. 223-237.

Lauritzen, S.-E., Løvlie, R., Moe, D., Østbye, E., 1990. Paleoclimate deduced from a multidisciplinary study of a halfmillion-year-old stalagmite from Rana, northern Norway. Quaternary Research. 34, 306-316.

Macintosh, N.W.G., 1952. Paintings in Beswick Creek Cave, Northern Territory. Oceania. 22, 256-274.

Martínez-Pillado, V., Aranburu, A., Arsuaga, J., Ruiz-Zapata, B., Gil-García, M., Stoll, H., Yusta, I., Iriarte, E., Carretero, J., Edwards, R., Cheng, H., 2014. Upper Pleistocene and Holocene palaeoenvironmental records in Cueva Mayor karst (Atapuerca, Spain) from different proxies: speleothem crystal fabrics, palynology, and archaeology. International Journal of Speleology. 43, 1-14.

Martínez-Pillado, V., Aranburu, A., Yusta, I., Stoll, H., Arsuaga, J.L., 2010. Clima y ocupaciones en la Galería de Estatuas (Atapuerca, Burgos) en los últimos 14.000 años: Relatos de una estalagmita. Munibe (AntropologiaArkeologia). 61, 89-102.

Perrette, Y., Delannoy, J.-J., Genty, D., Destombes, J.-L., Quinif, Y., 1997. Enregistrement de l'activité charbonnière dans les spéléothèmes de Choranche (Vercors, France). Proc. 12th Congr. of Speleol., Switzerland. 1, 61-64.

Petránek, J., Pouba, Z., 1951. Pokus o datování vývoje jaskyně Domice na základě studia tmavých zon v krápnících a sintru (Dating of the development of the Domica Cave, based on the study of the dark zones in the travertine formations). Sborník Ústředního Ústavu Geologického. 18, 245-272.

Slimak, L., 2004. Les dernières expressions du Moustérien entre Loire et Rhône (Thèse de doctorat). Université de Provence, Aix-en-Provence.

Solecki, R.S., 1979. Contemporary Kurdish winter-time inhabitants of Shanidar cave, Iraq. World Archaeology. 10, 318-330. 
Striebel, T., 1996. Höhlen im Gebiet der Stadt Blaubeuren. Presented at the VDHK-Jahrestreffen 1996, pp. 150-171.

Vandevelde, S., Brochier, J.É., Desachy, B., Petit, C., Slimak, L., 2018. Sooted concretions: A new Microchronological Tool for High Temporal Resolution Archaeology. Quaternary International. 474, 103-118.

Vandevelde, S., Brochier, J.É., Petit, C., Slimak, L., 2017. Establishment of occupation chronicles in Grotte Mandrin using sooted concretions: rethinking the Middle to Upper Paleolithic transition. Journal of Human Evolution. 112, 70-78.

Verheyden, S., Baele, J.-M., Keppens, E., Genty, D., Cattani, O., Cheng, H., Lawrence, E., Zhang, H., Van Strijdonck, M., Quinif, Y., 2006. The Proserpine Stalagmite (Han-sur-Lesse Cave, Belgium): Preliminary Environmental Interpretation of the Last 1,000 Years as Recorded in a Layered Speleothem. Geologica Belgica. 9, 245-256.

Watson, P.J., Yarnell, R.A., 1966. Archaeological and Paleoethnobotanical Investigations in Salts Cave, Mammoth Cave National Park, Kentucky. American Antiquity. 31, 842-849.

White, J.P., 1965. Archaeological Excavations in New Guinea: an Interim Report. The Journal of the Polynesian Society. 74, 40-56. 